
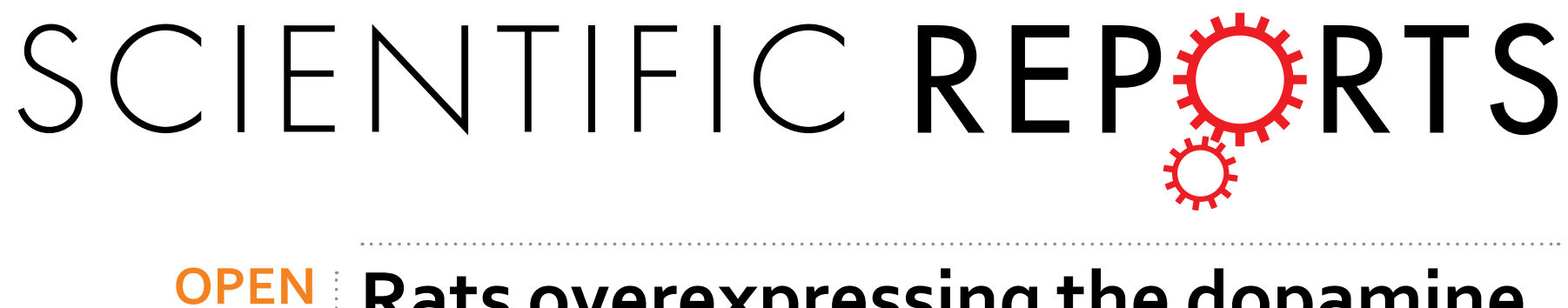

\title{
Rats overexpressing the dopamine transporter display behavioral and neurobiological abnormalities with \\ relevance to repetitive disorders
}

Received: 08 April 2016

Accepted: 18 November 2016

Published: 15 December 2016
Ravit Hadar ${ }^{1}$, Henriette Edemann-Callesen ${ }^{1,2}$, Claudia Reinel ${ }^{1}$, Franziska Wieske ${ }^{1}$, Mareike Voget ${ }^{1,2}$, Elena Popova ${ }^{3}$, Reinhard Sohr ${ }^{1}$, Yosef Avchalumov ${ }^{1}$, Josef Priller ${ }^{4}$, Christoph van Riesen ${ }^{5}$, Imke Puls ${ }^{6}$, Michael Bader ${ }^{3,7,8,9}$ \& Christine Winter ${ }^{1}$

The dopamine transporter (DAT) plays a pivotal role in maintaining optimal dopamine signaling. DAToveractivity has been linked to various neuropsychiatric disorders yet so far the direct pathological consequences of it has not been fully assessed. We here generated a transgenic rat model that via pronuclear microinjection overexpresses the DAT gene. Our results demonstrate that DAToverexpression induces multiple neurobiological effects that exceeded the expected alterations in the corticostriatal dopamine system. Furthermore, transgenic rats specifically exhibited behavioral and pharmaco-therapeutic profiles phenotypic of repetitive disorders. Together our findings suggest that the DAT rat model will constitute a valuable tool for further investigations into the pathological influence of DAT overexpression on neural systems relevant to neuropsychiatric disorders.

In the realm of neuroscience, preclinical studies promote our understanding of normal and pathological brain function as well as the development of new treatment strategies and are thus invaluable. This leads to ongoing innovation and generation of new model rodents. The development of new models is either done by the selection of existing phenomena or the rationale driven manipulation of a specific mechanism. The latter may comprise environmental, pharmacological or genetic manipulations. Genetic models start with addressing the etiology of the modeled disorder ${ }^{1}$ however they may only be considered complete upon meeting further construct, face and predictive validity criteria. In neuro-psychiatry etiology is mostly obscure, forcing scientists into testing the assumed etiology by comprehensively evaluating the consequences of the manipulation on aspects of brain and behavior known to be aberrant in the modeled disorder. Preclinical studies succumb to a classical differentiation between mice and rats, such that it is mostly mice, which provide rationale-driven genetic models whereas rats are devoted to behavioral and environmental manipulations, due to their superior social and behavioral repertoire. Clearly, the latter is the essence of psychiatric disorders, hence genetic rat models would ideally incorporate both aspects.

Interdisciplinary evidence suggests a pivotal role of the dopamine system and the corticostriatal circuitry in the pathology underlying repetitive disorders. Reduced tonic extracellular ${ }^{3}$, increased presynaptic ${ }^{4}$, and pharmacologically released intrasynaptic dopamine contents ${ }^{5}$ as well as increased dopamine receptor availability ${ }^{6}$, suggests an overactive dopamine transporter (DAT) ${ }^{7,8}$ in repetitive disorders, including Tourette syndrome (TS). Still, investigations into the direct consequences of DAT overexpression is underrepresented in preclinical studies with only very few models that allow insights into its relation to such neuropsychiatric disorders.

${ }^{1}$ Department of Psychiatry and Psychotherapy, University Hospital Carl Gustav Carus, Technische Universität, Dresden, Germany. ${ }^{2}$ International Graduate Program Medical Neurosciences, Charité Universitätsmedizin Berlin, Germany. ${ }^{3}$ Max-Delbrück-Center for Molecular Medicine, Berlin, Germany. ${ }^{4}$ Department of Neuropsychiatry and Laboratory of Molecular Psychiatry, Charité Universitätsmedizin Berlin, Cluster of Excellence NeuroCure, BIH and DZNE, Berlin, Germany. ${ }^{5}$ Department of Neurology, Charité Universitätsmedizin Berlin, Germany. ${ }^{6} \mathrm{Klinik}$ für Psychiatrie und Psychotherapie, Charité Universit, Berlin, Germany. ${ }^{7}$ Department of Endocrinology, Charité Universitätsmedizin Berlin, Germany. ${ }^{8}$ Institute for Biology, University of Lübeck, Lübeck, Germany. ${ }^{9}$ Department of Physiology and Biophysics, Federal University of Minas Gerais, Belo Horizonte, Brazil. Correspondence and requests for materials should be addressed to C.W. (email: christine.winter@uniklinikum-dresden.de) 
a

5 flanked DNA-region
and NSE-promotor

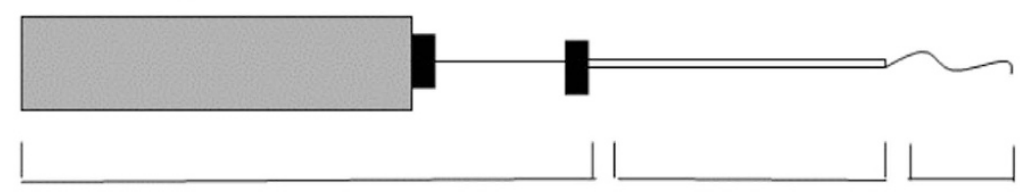

$\begin{array}{lll}\text { NSE rat } & \text { mDAT } & \text { SV40 }\end{array}$

b

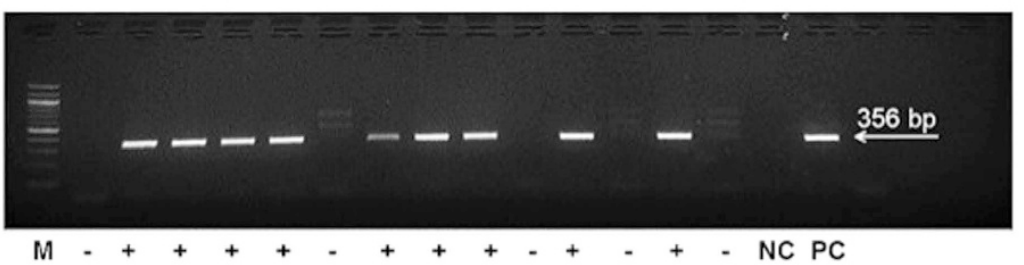

C

wt
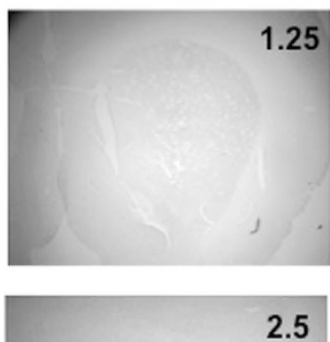

2.5

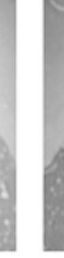

\section{DAT-tg}
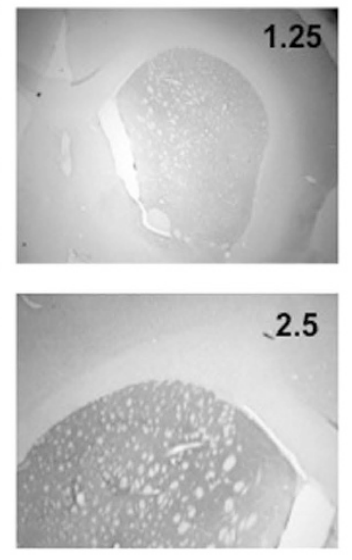

d

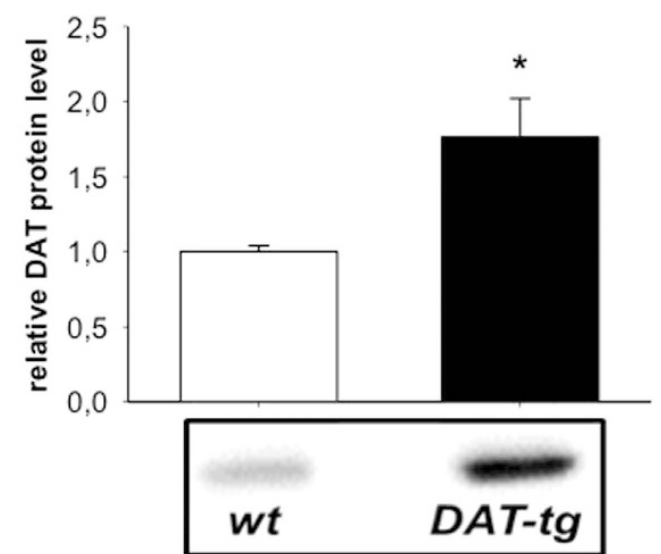

Figure 1. Generation of DAT-tg rats. (a) Schematic representation of the 4-kb DNA fragment used for the generation of the DAT transgenic rats. E1/2= exon $1 / 2$, and $\mathrm{I} 1=$ intron 1 of NSE, $\mathrm{mDAT}=$ murine DAT sequence, $\mathrm{SV} 40=$ Simian virus 40. (b) Representative DAT PCR products from $w t(-)$ and DAT-tg $(+)$ rats. $\mathrm{M}=$ marker, $\mathrm{NC}=$ negative control, $\mathrm{PC}=$ positive control, transgenic band $=356 \mathrm{bp}$. One founder line was used for the study. Here one litter from this generation is shown. (c) Representative coronal sections of immunohistochemical stain of DAT expression for wt (left) and DAT-tg (right). (d) DAT Western blot analysis of striatal tissue from $w t(\mathrm{n}=5)$ and DAT-tg rats $(\mathrm{n}=10)$.

On this basis we created a transgenic rat model that via pronuclear microinjection overexpresses the DAT gene (Fig. 1). Neurobiological and behavioral studies were conducted on adult male hemizygous DAT-transgenic rats (DAT-tg) ubiquitously overexpressing DAT in the corticostriatal and associated networks.

\section{Results}

DAT and DRD1/2 receptor expression. Western blot and qPCR were performed in order to assess the protein and mRNA expression levels of the dopamine transporter (DAT). qPCR was conducted to assess mRNA expression levels of the dopamine receptor 1 (DRD1), and dopamine receptor 2 (DRD2). Western blots showed that in comparison to $w t$ rats, DAT-tg rats exhibited increased striatal protein-levels of the DAT transporter (striatum: $\mathrm{T}=-2.171, \mathrm{p}=0.05$ ) (Fig. $1 \mathrm{~d}$ ). $\mathrm{qPCR}$ showed that in comparison to $w t$ rats DAT-tg rats exhibited significantly increased DAT mRNA levels in the following areas: medial prefrontal cortex $(\mathrm{mPFC}(\mathrm{T}=-2.588$, $\mathrm{p}=0.023)$ ), orbitofrontal cortex $(\mathrm{OFC}(\mathrm{T}=9.161, \mathrm{p}=0.000))$, nucleus accumbens $(\mathrm{Nacc}(\mathrm{T}=-2.755$, $\mathrm{p}=0.016))$, caudate putamen $(\mathrm{CPu}(\mathrm{T}=-8.337, \mathrm{p}=0.000))$, globus pallidus $(\mathrm{GP}(\mathrm{T}=-4.579, \mathrm{p}=0.000))$, 
a

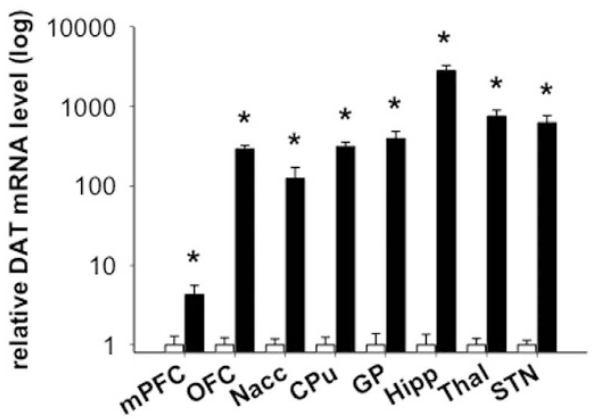

b

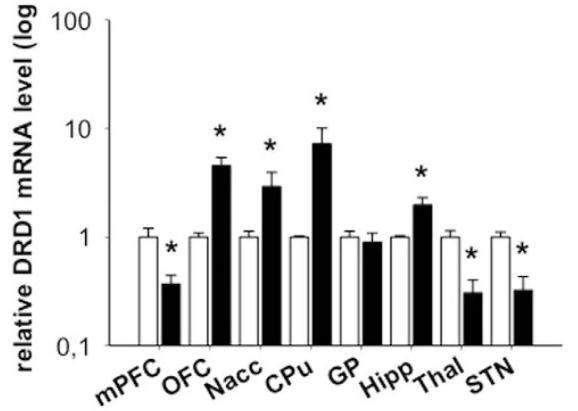

C
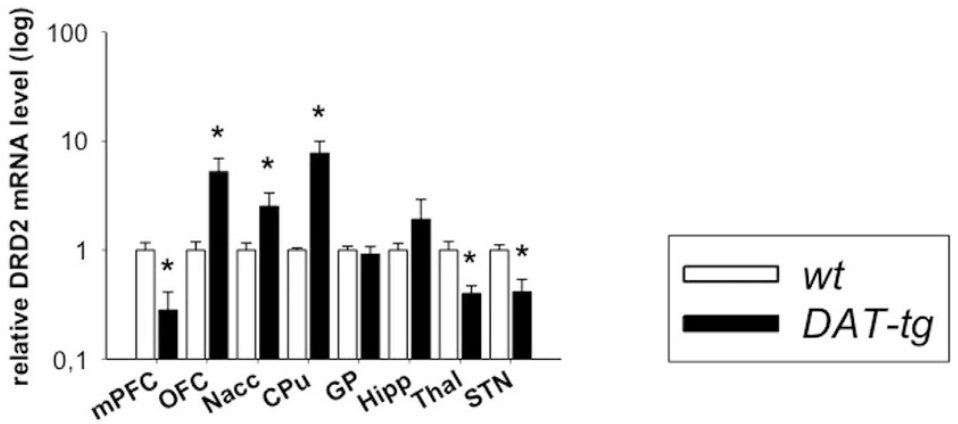

Figure 2. DAT and DRD1/2 receptor expression. (a) DAT $\mathrm{qPCR}$ analysis of corticostriatal and associated network regions in $w t(\mathrm{n}=8)$ and DAT-tg rats $(\mathrm{n}=7)$. (b) Dopamine receptor 1 (DRD1) and (c) Dopamine receptor 2 (DRD2) qPCR analysis of corticostriatal and associated network regions in $w t(\mathrm{n}=8)$ and DAT-tg rats $(\mathrm{n}=7)$. mPFC: medial prefrontal cortex, OFC: orbitofrontal cortex, Nacc: nucleus accumbens, CPu: caudate putamen, GP: globus pallidus, Hipp: hippocampus, Thal: dorsomedial thalamus, STN: subthalamic nucleus. All data are means \pm s.e.m. Asterisk $\left.{ }^{*}\right)$ indicates significant difference to $w t$ rats with $\mathrm{p}<0.05$.

hippocampus (Hipp (T-6.463, $\mathrm{p}=0.001)$ ), thalamus (Thal $(\mathrm{T}=-5.410, \mathrm{p}=0.000)$ ), and subthalamic nucleus $(\mathrm{STN}(\mathrm{T}=-4,589, \mathrm{p}=0.000))$ (Fig. 2a). Further, DAT- $\operatorname{tg}$ rats exhibited increased DRD1 mRNA levels in the OFC $(\mathrm{T}=-3.534, \mathrm{p}=0.000), \operatorname{Nacc}(\mathrm{T}=-2.136, \mathrm{p}=0.029), \mathrm{CPu}(\mathrm{T}=-6.217, \mathrm{p}=0.036)$ and Hipp $(\mathrm{T}=-3.089$, $\mathrm{p}=0.009)$ and decreased DRD1 mRNA levels in the $\mathrm{mPFC}(\mathrm{T}=2.756, \mathrm{p}=0.016)$, Thal $(\mathrm{T}=3.812, \mathrm{p}=0.002)$ and STN $(\mathrm{T}=4.332, \mathrm{p}=0.000)$ (Fig. $2 \mathrm{~b})$. In a similar fashion, DRD2 receptors were upregulated in the OFC $(\mathrm{T}=-$ $2.610, \mathrm{p}=0.022)$, NAcc $(\mathrm{T}=-1.917, \mathrm{p}=0.029)$ and $\mathrm{CPu}(\mathrm{T}=-3.252, \mathrm{p}=0.006)$ whereas levels were downregulated in the $\mathrm{mPFC}(\mathrm{T}=3.246, \mathrm{p}=0.006)$, Thal $(\mathrm{T}=2.646, \mathrm{p}=0.02)$ and $\mathrm{STN}(\mathrm{T}=3.414, \mathrm{p}=0.005)$ (Fig. 2c).

Neurotransmitter contents and compensatory mechanisms. Post mortem HPLC was conducted to assess neurochemical contents of different neurotransmitter system. DAT-tg rats exhibited a decrease in tissue dopamine contents in the OFC $(\mathrm{T}=-7.504, \mathrm{p}=0.000)$, Nacc $(\mathrm{T}=-13,726, \mathrm{p}=0.000)$ and $\mathrm{CPu}(\mathrm{T}=-14.611$, $\mathrm{p}=0.000)$, whereas an increase in dopamine was seen in the Hipp $(\mathrm{T}=2.617, \mathrm{p}=0.020)$ and STN $(\mathrm{T}=2.414$, $\mathrm{p}=0.029$ ). With regards to metabolites and turnorver, DAT-tg rats exhibited increased DOPAC contents and dopamine turnover (DOPAC/dopamine) in the $\mathrm{mPFC}(\mathrm{DOPAC}$ : $\mathrm{T}=4.255, \mathrm{p}=0.000$; turnover: $\mathrm{T}=2.916$, $\mathrm{p}=0.011)$, OFC $(\mathrm{T}=3.225, \mathrm{p}=0.006$; turnover: $\mathrm{T}=13.467, \mathrm{p}=0.000)$, Nacc $(\mathrm{T}=4.391, \mathrm{p}=0.000$; turnover: $\mathrm{T}=7.542, \mathrm{p}=0.000), \mathrm{CPu}(\mathrm{T}=9.134, \mathrm{p}=0.000$; turnover: $\mathrm{T}=19.314, \mathrm{p}=0.000), \mathrm{GP}(\mathrm{T}=6.177, \mathrm{p}=0.000$; turnover: $\mathrm{T}=7.417, \mathrm{p}=0.000)$, Hipp $(\mathrm{T}=5.884, \mathrm{p}=0.000$; turnover: $\mathrm{T}=1.35, \mathrm{p}=0.022)$, Thal $(\mathrm{T}=4.009,0.001$; turnover: $\mathrm{T}=1.505, \mathrm{p}=0.001)$ and $\operatorname{STN}(\mathrm{T}=4.503, \mathrm{p}=0.000$; turnover: $\mathrm{T}=2.962, \mathrm{p}=0.010)($ Fig. $3 \mathrm{a}-\mathrm{c})$. For glutamate, DAT-tg rats exhibited increased contents in the $\mathrm{CPu}(\mathrm{T}=2.701, \mathrm{p}=0.016), \mathrm{GP}(\mathrm{T}=4.934, \mathrm{p}=0.000)$ and STN $(\mathrm{T}=4.113, \mathrm{p}=0.000)$ whereas a decrement was found in the thalamus $(\mathrm{T}=-4.574, \mathrm{p}=0.000)$. With respect to GABA, DAT-tg rats exhibited decreased contents in the Nacc $(\mathrm{T}=-2.665, \mathrm{p}=0.018)$ and GP 


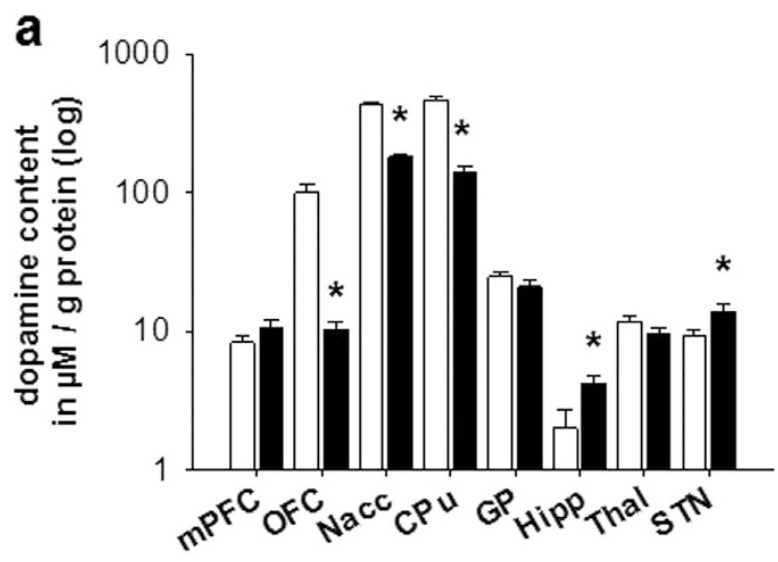

b

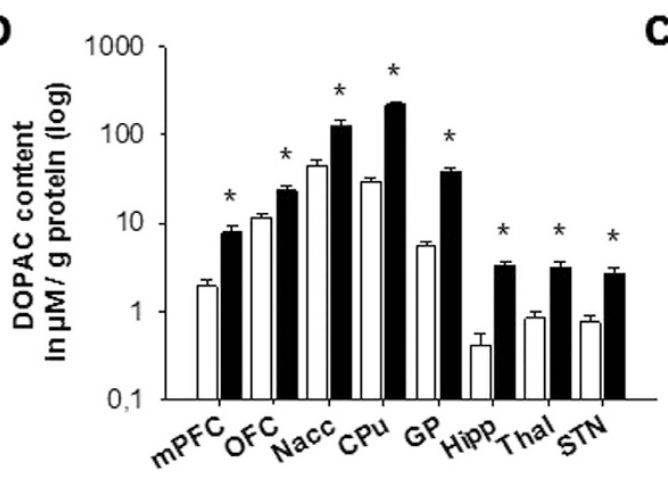

d

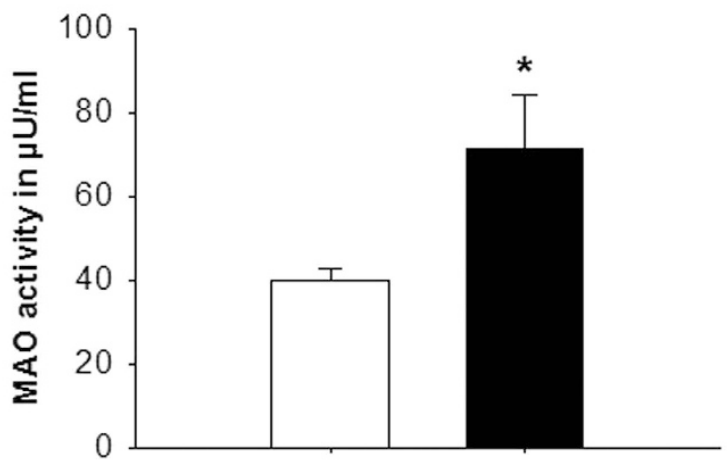

C

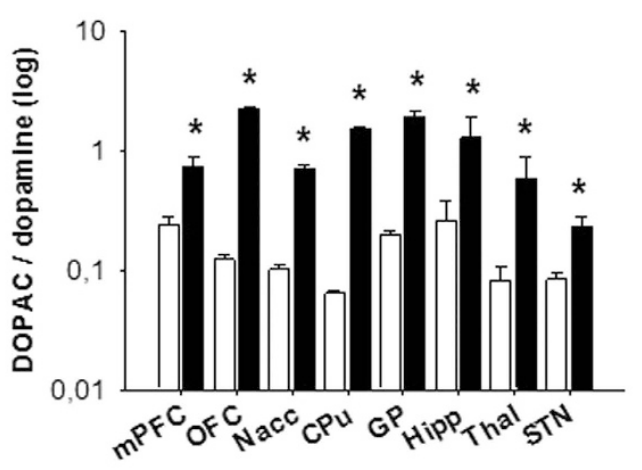

Figure 3. Neurotransmitter contents and compensatory mechanisms. Post mortem tissue (a) dopamine and (b) DOPAC contents as well as (c) dopamine turnover in corticostriatal and associated network regions in $w t(\mathrm{n}=7)$ and DAT-tg rats $(\mathrm{n}=10)$. mPFC: medial prefrontal cortex, OFC: orbitofrontal cortex, Nacc: nucleus accumbens, $\mathrm{CPu}$ : caudate putamen, GP: globus pallidus, Hipp: hippocampus, Thal: dorsomedial thalamus, STN: subthalamic nucleus. (d) Monoamine oxidase (MAO) activity of striatal tissues in $w t(\mathrm{n}=8)$ and $D A T$ - $t g$ rats $(\mathrm{n}=7)$. All data are means \pm s.e.m. Asterisk $\left.{ }^{*}\right)$ indicates significant difference to $w t$ rats with $\mathrm{p}<0.05$.

$(\mathrm{T}=-2.231, \mathrm{p}=0.041)$ and increased contents in the $\mathrm{mPFC}(\mathrm{T}=2.962, \mathrm{p}=0.009), \mathrm{OFC}(\mathrm{T}=3.161, \mathrm{p}=0.006)$ and $\mathrm{CPu}(\mathrm{T}=3.449, \mathrm{p}=0.004)$ (Fig. 4).

To investigate for possible compensatory mechanisms, monoamine oxidase (MAO) activity was assessed in striatal tissues. DAT-tg rats here exhibited a significant increase in total MAO activity as opposed to the $w t$ rats $(\mathrm{T}=-2.470, \mathrm{p}=0.028)$ (Fig. 3d).

Oscillatory activity. Oscillatory activity within the vmPFC, Nacc and STN was investigated via in vivo electrophysiological recording. The assessed frequency bands included: theta $(4-8 \mathrm{~Hz})$, alpha $(8-12 \mathrm{~Hz})$, beta $(12-30 \mathrm{~Hz})$, and gamma $(30-100 \mathrm{~Hz})$. Results show that in comparison to $w t$ rats DAT-tg rats exhibited increased alpha, beta and gamma activity within the STN (alpha: $\mathrm{T}=-8.667, \mathrm{p}=0.000$; beta: $\mathrm{T}=-8.972, \mathrm{p}=0.000$; gamma: $\mathrm{T}=-2.781$, $\mathrm{p}=0.006$ ) as well as increased beta and gamma activity within the $\mathrm{mPFC}$ (beta: $\mathrm{T}=-6.701, \mathrm{p}=0.000$; gamma: $\mathrm{T}=-3.389, \mathrm{p}=0.000$ ) and Nacc (beta: $\mathrm{T}=-3.723, \mathrm{p}=0.000$; gamma: $\mathrm{T}=-2.594, \mathrm{p}=0.01$ ) (Fig. 5a). 


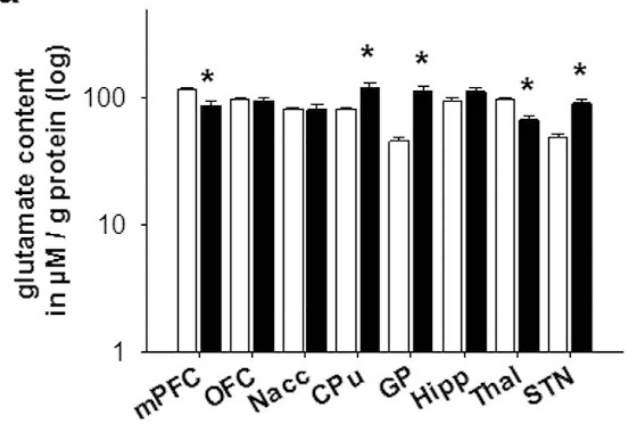

b

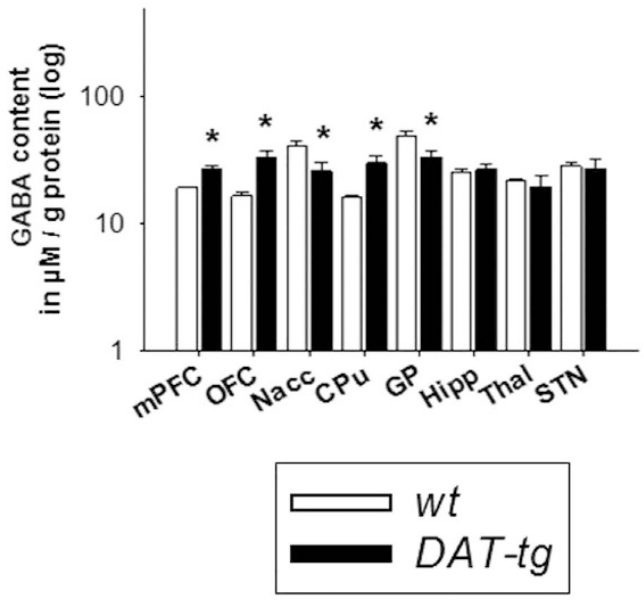

Figure 4. Glutamate and GABA contents. Neurochemical contents were examined in $w t(\mathrm{n}=7)$ and DAT-tg rats $(\mathrm{n}=10)$. Glutamate and GABA were measured in the medial prefrontal cortex (mPFC), orbitofrontal cortex (OFC), nucleus accumbens (Nacc), caudate putamen ( $\mathrm{CPu}$ ), globus pallidus (GP), hippocampus (Hipp), dorsomedial thalamus (Thal), and subthalamic nucleus (STN). All data are means \pm s.e.m. Asterisk $\left({ }^{*}\right)$ indicates significant difference to $w t$ rats with $\mathrm{p}<0.05$.

Immunostaining. Immunostaining of parvalbumin expressing (PV)+ interneurons, c-Fos expressing nuclei and Ki67 expressing cells was conducted to investigate for possible cellular changes reflecting altered network activity. Results show that DAT-tg rats exhibited a significant reduction of $\mathrm{PV}+$ cells specifically in the $\mathrm{CPu}$ as opposed to $w t$ rats $(\mathrm{T}=3.228, \mathrm{p}=0.004)$ (Fig. $5 \mathrm{~b}$ ). Further, DAT-tg rats exhibited a significant increase in cFos expressing cells specifically in the OFC as compared to $w t$ rats $(\mathrm{T}=-2.884, \mathrm{p}=0.011)$ (Fig. $5 \mathrm{c})$. No significant difference was found for Ki67 expression between DAT-tg and $w t$ rats (Fig. 5d).

Structural analysis of brain volumes. The whole brain volume and the volumes of the mPFC, Hipp, and $\mathrm{CPu}$ were assessed using structural MRI. DAT-tg rats exhibited a significant increase in Hipp volumes as compared to the $w t$ rats $(\mathrm{T}=-3.326, \mathrm{p}=0.01)$ alongside unaltered whole brain volumes (Fig. $6 \mathrm{a})$. NeuN immunostaining further revealed no difference between DAT- $t g$ and $w t$ rats (Fig. 6b).

General behavioral assessment. Wt and DAT-tg rats were weighed across lifespan and body weights of $D A T$ - $t g$ rats were analyzed relative to body weight of age-matched $w t$ rats. T-Test revealed $D A T$ - $t g$ rats to have significantly decreased body weights in comparison to $w t$ rats across lifespan $(\mathrm{T}=6.801, \mathrm{P}=0.000)$ (Fig. 7a). Figure $7 \mathrm{~b}$ locomotion was analyzed as the total distance travelled on an open field over $30 \mathrm{~min}$. T-Test revealed $D A T$ - $t g$ rats to travel significantly less than $w t$ rats $(\mathrm{T}=5.745, \mathrm{P}=0.001)$ (Fig. $7 \mathrm{~b}$ ). Figure $7 \mathrm{a}$ to study repetitive behavior upon stress-exposure, rats were exposed to unpredictable acoustic stimuli. T-test revealed DAT-tg rats to show a tendency towards more grooming $(\mathrm{T}=-2.070, \mathrm{P}=0.063)$ when compared to $w t$ rats, but no significant increment in the number of whole body shakes $(\mathrm{T}=-1.527, \mathrm{P}=0.156)$ (Fig. $7 \mathrm{c}$ ).

In the prepulse inhibition (PPI) paradigm, DAT-tg rats showed normal sensorimotor gating when compared to $w t$ rats such that they expressed an unaltered suppression of the acoustic startle reflex (ASR) following acoustic stimuli of $69 \mathrm{db}, 73 \mathrm{db}$, and $81 \mathrm{db}$. However DAT-tg rats did show increased overall ASR reflecting hyper-arousal $(\mathrm{T}=-2.449, \mathrm{P}=0.024)$ (Fig. $7 \mathrm{~d}$ ). In the elevated-plus-maze and the forced swim test, no difference were found between DAT-tg rats and $w t$ rats (Fig. 7e,f). In the sucrose consumption test DAT-tg rats showed when compared to $w t$ rats a tendency to increased anhedonia as expressed in a reduced consumption of sweetened condense milk $(\mathrm{T}=1.659, \mathrm{P}=0.071)$ (Fig. $7 \mathrm{~g})$.

Repetitive behavior analysis. Repetitive behavior was assessed following the application of amphetamine $(0.5 \mathrm{mg} / \mathrm{kg}, 2.0 \mathrm{mg} / \mathrm{kg}$, and $5.0 \mathrm{mg} / \mathrm{kg}$ body weight $(\mathrm{BW}))$ and saline over three consecutive days. To diminish the possibility of amphetamine-sensitization, dosages were applied in a randomized fashion. The assessed behavior included: no locomotion, locomotion, excessive rearing and sniffing as well as oral stereotypy and head movements. Administration of $0.5 \mathrm{mg} / \mathrm{kg}$ amphetamine was ineffective in both strains and administration of $5.0 \mathrm{mg} / \mathrm{kg}$ amphetamine induced repetitive behavior in both, $w t$ and DAT- $t g$ rats. Upon administration of $2.0 \mathrm{mg} / \mathrm{kg}$ amphetamine, DAT-tg rats exhibited a significant increase in repetitive oral movements $(\mathrm{T}=-3,545, \mathrm{p}=0.003)$, which effectively emerged $80-120 \mathrm{~min}$ after injection, whereas $w t$ rats exhibited hyper-locomotion throughout the observation period $(\mathrm{T}=4,718, \mathrm{P}=0.000)$ (Fig. $8 \mathrm{a}$ ).

The effect of clonidine $(0.01 \mathrm{mg} / \mathrm{kg} \mathrm{BW})$ and fluoxetine $(20 \mathrm{mg} / \mathrm{kg} \mathrm{BW})$ versus saline on amphetamine $(2 \mathrm{mg} / \mathrm{kg}$ BW) -induced behavior was assessed with respect to general movement and oral stereotypy. Same animals were exposed to drug administrations over a period of three consecutive days, with dosages applied in a randomized fashion. For the effect of clonidine on oral stereotypy, ANOVA revealed a significant effect for the factor 
a
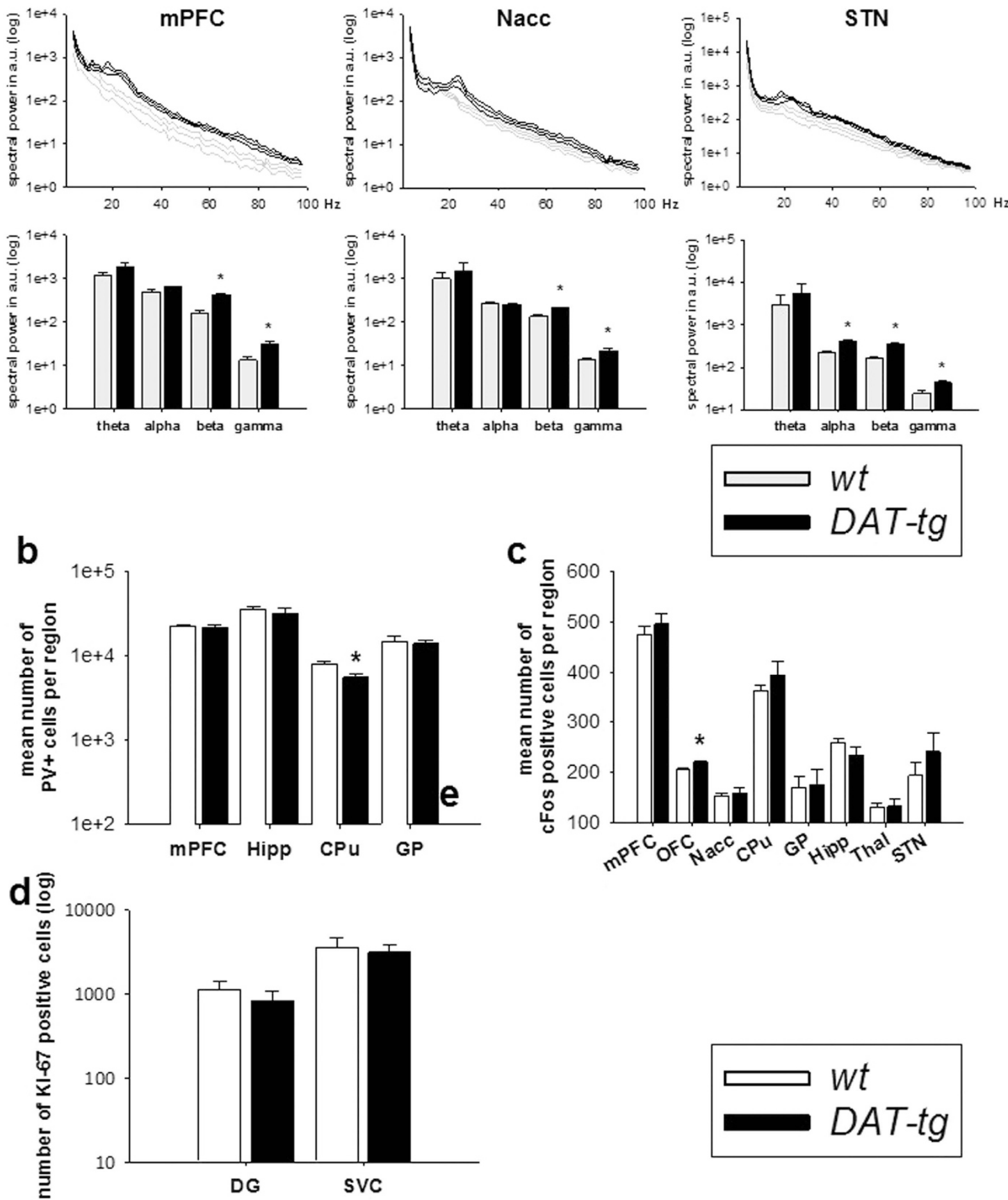

Figure 5. Oscillatory activity and immunostaining. (a) Oscillatory activity of the vmPFC, Nacc and STN in $w t(\mathrm{n}=5)$ and $D A T$ - $\operatorname{tg}$ rats $(\mathrm{n}=7)$. Upper panel shows entire frequency range, lower panel shows mean values for the frequency bands: theta $(4-8 \mathrm{~Hz})$, alpha $(8-12 \mathrm{~Hz})$, beta $(12-30 \mathrm{~Hz})$, and gamma $(30-100 \mathrm{~Hz})$. (b) Immunohistochemical cell counts of parvalbumin expressing $(\mathrm{PV}+)$ cells of the medial prefrontal cortex (mPFC), hippocampus (Hipp), caudate putamen $(\mathrm{CPu})$ and globus pallidus $(\mathrm{GP})$ in $w t(\mathrm{n}=12)$ and $D A T$ - $\operatorname{tg}$ rats $(\mathrm{n}=11)$. (c) c-Fos expressing cells on representative slices of the mPFC, orbitofrontal cortex (OFC), nucleus accumbens (Nacc), CPu, GP, Hipp, thalamus (Thal) and subthalamic nucleus (STN) in $w t(\mathrm{n}=8)$ and DAT-tg rats $(n=8)$. (d) Immunohistochemical cell counts of Ki67 expressing cells in the neurogenic zones of the hippocampus (dentate gyrus, DG) and the subventricular zone (SVZ) in $w t(\mathrm{n}=9)$ and DAT-tg rats $(\mathrm{n}=9)$. 
a
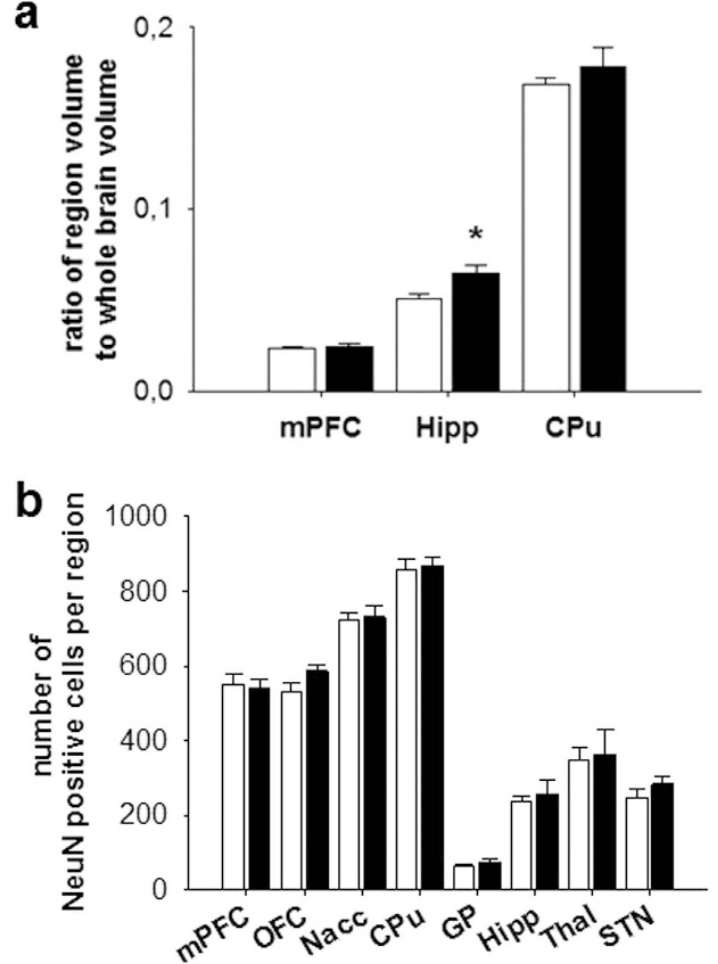

Figure 6. Structural analysis. (a) Volumes of the medial prefrontal cortex (mPFC), hippocampus (Hipp) and caudate putamen $(\mathrm{CPu})$ relative to whole brain volumes as derived from MRI scans in $w t(\mathrm{n}=6)$ and DAT-tg rats $(\mathrm{n}=4)$. (b) NeuN expressing cells on representative slices of the mPFC, orbitofrontal cortex (OFC), nucleus accumbens (Nacc), CPu, globus pallidus (GP), Hipp, thalamus (Thal) and subthalamic nucleus (STN) in $w t$ $(\mathrm{n}=7)$ and DAT-tg rats $(\mathrm{n}=5)$. All data are means \pm s.e.m. Asterisk $\left.{ }^{*}\right)$ indicates significant difference to $w t$ rats $(\mathrm{p}<0.05)$.

phenotype $(\mathrm{F}=6,598, \mathrm{p}=0.019)$ and a significant interaction $(\mathrm{F}=6.887, \mathrm{p}=0.018)$. Subsequent post hoc analysis revealed that untreated DAT-tg rats exhibited significantly more repetitive behavior than untreated $w t$ rats $(\mathrm{p}<0.05)$ and that clonidine significantly reduced repetitive behavior in DAT-tg rats $(\mathrm{p}<0.05)$. With regards to the effects of clonidine on locomotion, no significant effect was found. The effect of fluoxetine on oral stereotypy showed a significant effect for the factors phenotype $(\mathrm{F}=27.061, \mathrm{p}=0.000)$ and treatment $(\mathrm{F}=10.382, \mathrm{p}=0.006)$ with $D A T$ - $t g$ rats displaying significantly more repetitive behavior than $w t$ rats and fluoxetine reducing it in both, $w t$ and DAT-tg rats. With regards to the effect of fluoxetine on locomotion, a significant effect of treatment was found $(\mathrm{F}=15.127, \mathrm{p}=0.001)$ (Fig. $8 \mathrm{~b}$ ) such that fluoxetine reduced locomotion in both, wt and DAT-tg rats.

The effect of quinpirole $(0.5 \mathrm{mg} / \mathrm{kg} \mathrm{BW})$ and saline on compulsive checking and grooming was assessed using the following groups: $w t+$ saline, $w t+$ quinpirole, $D A T-t g+$ saline, $D A T$ - $t g+$ quinpirole. For compulsive checking, a significant effect was found for phenotype $(\mathrm{F}=11.464, \mathrm{p}=0.003)$ as well as a significant interaction across the factors phenotype and treatment. $(\mathrm{F}=5.283, \mathrm{p}=0.032)$. Subsequent post hoc analysis revealed that quinpirole treated $w t$ rats exhibited significantly more compulsive checking behavior as compared to untreated $w t(\mathrm{p}<0.05)$ and quinpirole treated $D A T$ - $t g$ rats $(\mathrm{p}<0.05)$. For grooming, a significant effect was found for both factors (phenotype: $\mathrm{F}=22.960, \mathrm{p}=0.001$; treatment: $\mathrm{F}=17.091, \mathrm{p}=0.003)$ as well as a significant interaction $(\mathrm{F}=21.278$, $\mathrm{p}=0.002$ ) (Fig. 8b). Figure $8 \mathrm{c}$ following up on these effects, post hoc analysis revealed that in DAT-tg but not $w t$ $(\mathrm{p}<0.05)$ quinpirole significantly increased grooming when compared to saline conditions $(\mathrm{p}<0.05)$.

\section{Discussion}

Our results show that overexpression of the DAT induces multiple neurobiological and behavioral deficits that have also been observed in repetitive disorders.

Involuntary repetitive movements have shown to worsen under stress and upon amphetamine challenge. Such accentuated susceptibility to amphetamine has been reported for TS and differentiates this condition from obsessive-compulsive disorders (OCD), a further disorder belonging to the repetitive spectrum ${ }^{9}$. In terms of pharmacotherapy, the alpha-adrenergic and imidazoline receptor agonist $\alpha$-clonidine serves as first line treatment due to its efficacy and tolerability ${ }^{10,11}$.

In rats, a typical expression of repetitive movements is grooming ${ }^{12}$. We here report, that DAT-tg rats showed increased grooming upon stress exposure. Upon d-amphetamine administration, both $w t$ and DAT-tg rats developed repetitive behavior ${ }^{13}$. However, $D A T$ - $t g$ rats developed repetitive behavior already at amphetamine dosages ineffective in $w t$ rats suggesting a susceptibility to amphetamine. This low-dose amphetamine induced repetitive behavior manifested over time with maximal expression 80-120 min after drug administration. It 
a

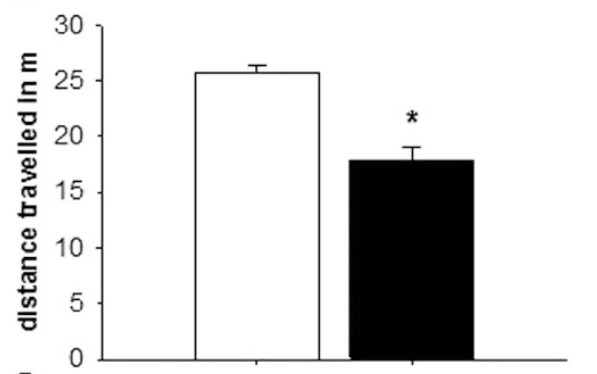

C
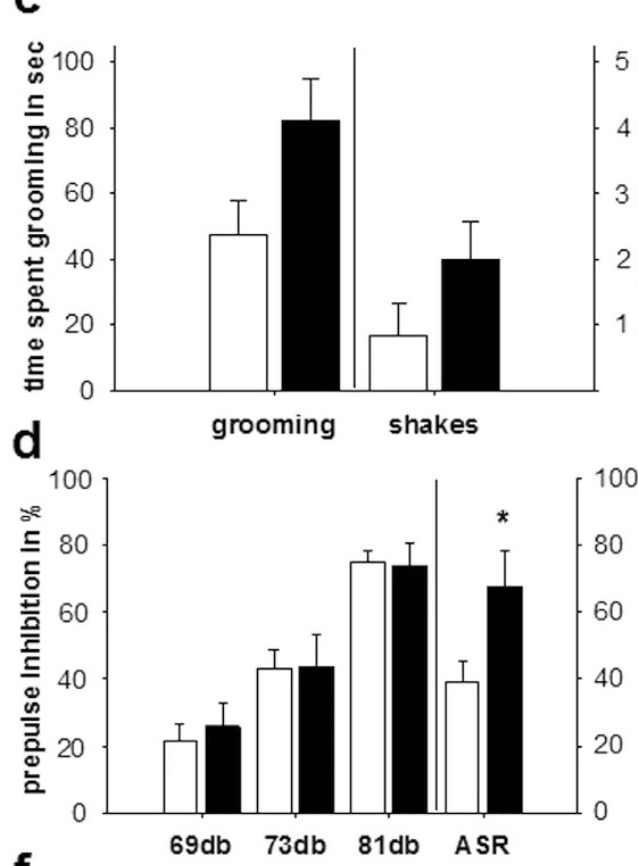

f

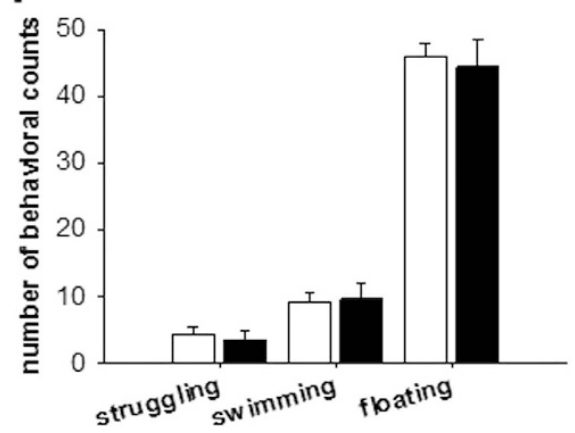

b
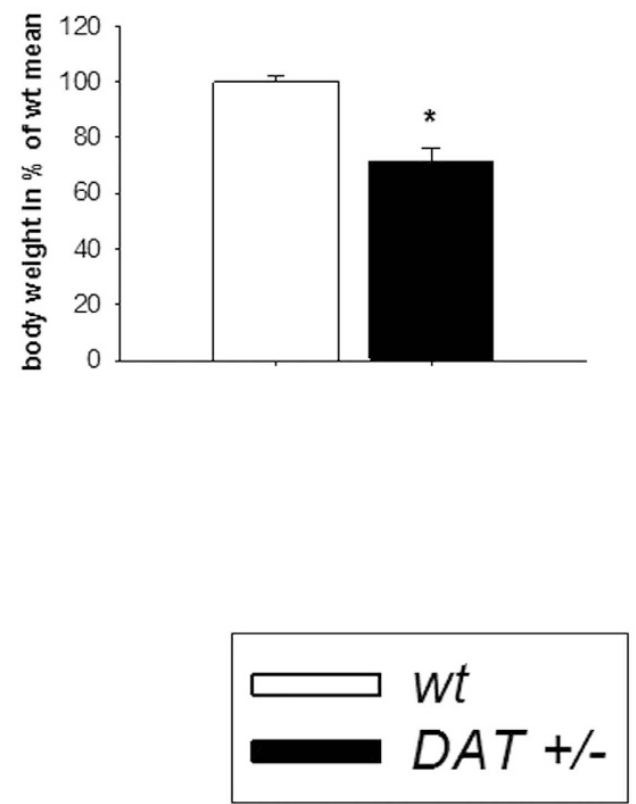

e

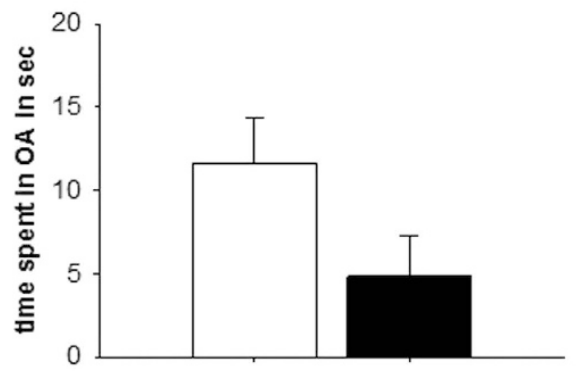

g

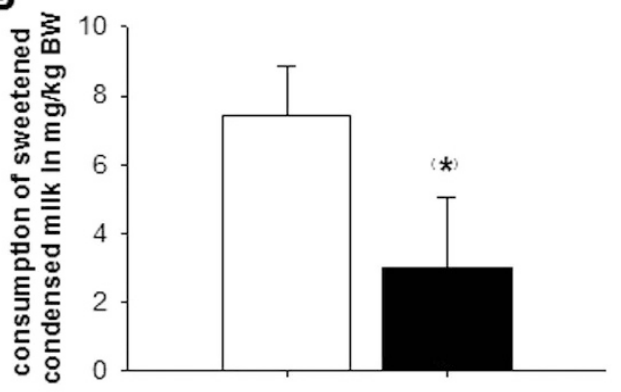

Figure 7. General behavioral assessment. General assessment of hemizygote $D A T$ transgenic (DAT-tg) rats in comparison to $w t$ rats. (a) $w t$ and DAT-tg rats were weighed across lifespan. Body weight of DAT-tg rats was analyzed relative to body weight of age-matched $w t$ rats. (b) Locomotion was analyzed as the total distance travelled on an open field over $30 \mathrm{~min}$ in each $\mathrm{n}=10 \mathrm{wt}$ and DAT-tg rats. (c) To study repetitive behavior upon stress-exposure, each $\mathrm{n}=7 \mathrm{wt}$ and $D A T$ - $\operatorname{tg}$ rats were placed within the chambers used for prepulse inhibition (PPI) test and exposed to unpredictable acoustic stimuli. (d) Sensorimotor gating and arousal was analyzed in the PPI paradigm in $w t(n=14)$ and DAT- $t g$ rats $(\mathrm{n}=7)$. (e) Anxious behavior was measured in the elevated-plus-maze in $w t(\mathrm{n}=15)$ and DAT-tg rats $(\mathrm{n}=6)$. (f) In the forced swim test, the amount of time spent on struggling, swimming and floating was analyzed in $w t(\mathrm{n}=14)$ and DAT-tg rats $(\mathrm{n}=7)$. $(\mathrm{g})$ In the sucrose consumption test the amount of sweetened condense milk consumed relative to body weight (BW) was measured in $w t$ rats $(\mathrm{n}=16)$ and DAT-tg rats $(\mathrm{n}=7)$. All data are given as mean \pm s.e.m. Asterisks $\left(^{\star}\right)$ indicates significant difference between groups with $\mathrm{p}<0.05$.

consisted of fragmented grooming patterns of face and paws that rarely continued into a full-body grooming syntax, and was associated with a typical motor confinement. Interestingly, this particular behavior was also 
a
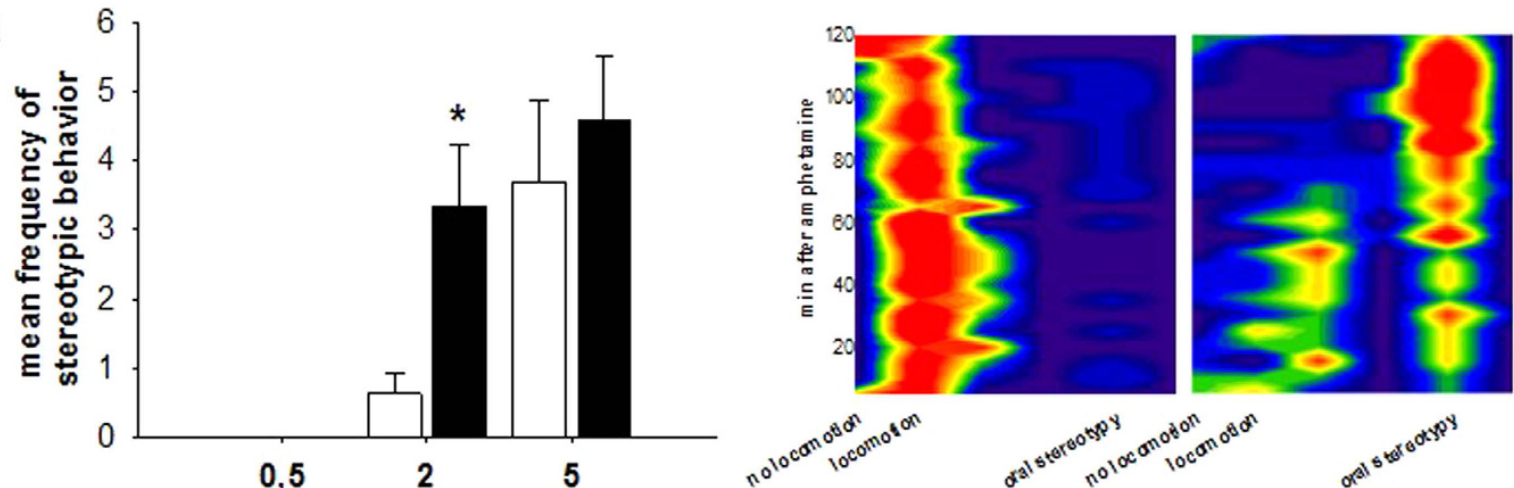

0,5

2
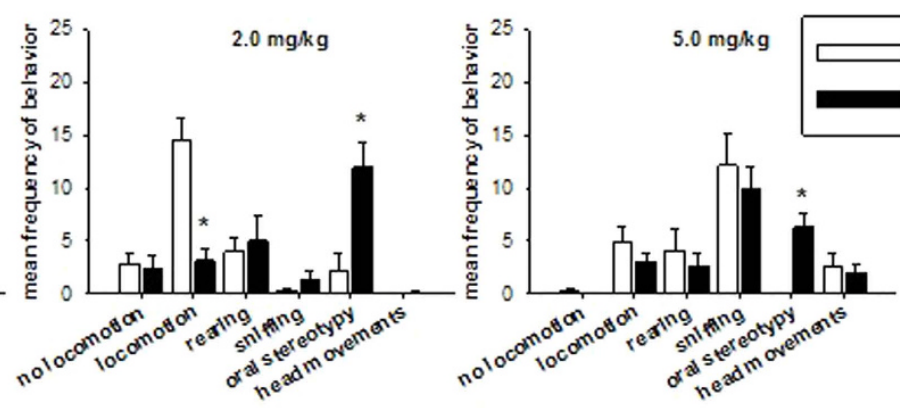

b
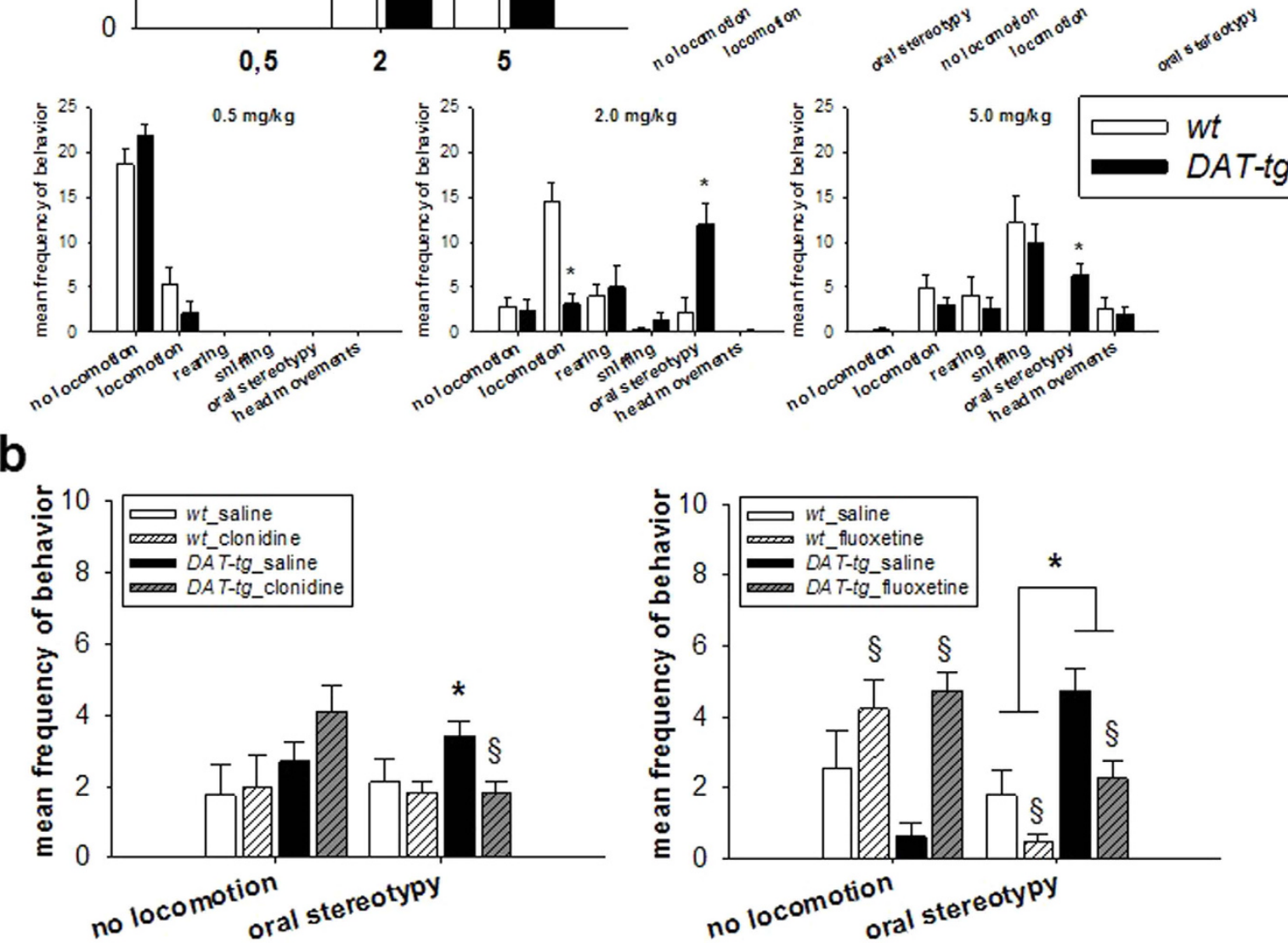

C

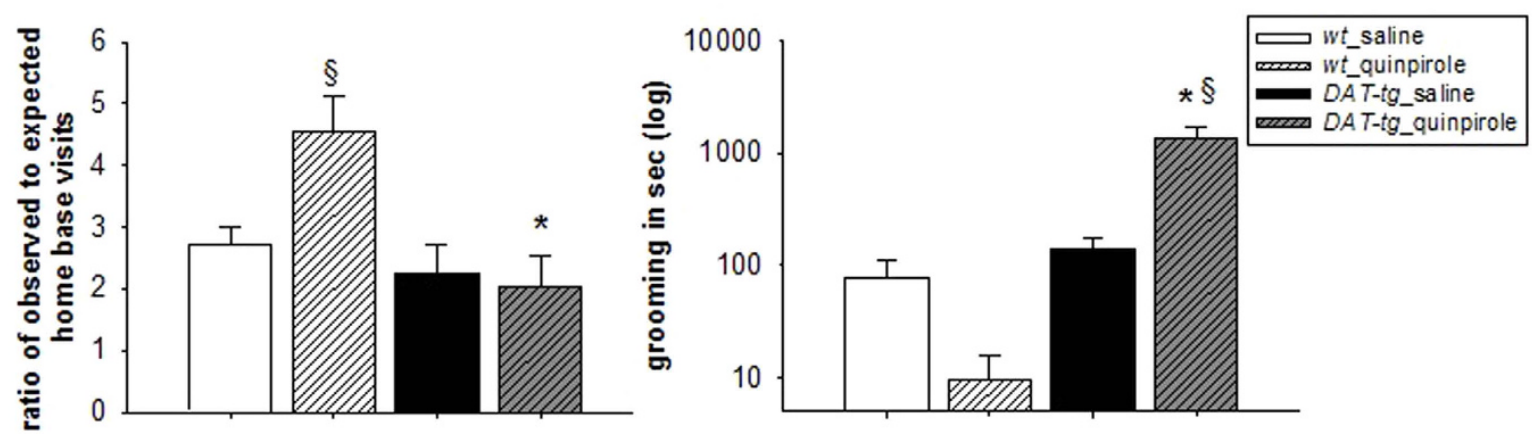

Figure 8. Repetitive behavior analysis. (a) Upper panel left: Repetitive behavior induced by d-amphetamine $(0.5,2.0$ and $5.0 \mathrm{mg}$ per kg body weight (BW)). Upper panel right: In DAT-tg rats, repetitive behavior evolves 80-120 min after d-amphetamine ( $2 \mathrm{mg} / \mathrm{kg} \mathrm{BW})$. The $w t$ rats display hyperlocomotion throughout the period (120 min). Hot colors (red) indicate presence and cold colors (blue) absence of behavior. Lower panel: dosedependent effects of d-amphetamine. (b) Clonidine effects (left) and fluoxetine (right) on locomotion and oral stereotypy following d-amphetamine $(2 \mathrm{mg} / \mathrm{kg} \mathrm{BW})$ in $w t$ (clonidine: $\mathrm{n}=10$; fluoxetine: $\mathrm{n}=9$ ) and DAT- $\operatorname{tg}$ rats (clonidine: $\mathrm{n}=9$; fluoxetine. $\mathrm{n}=8$ ). (c) Effects of quinpirole (QNP) on compulsive checking (left) and grooming behavior (right) in $w t(\mathrm{n}=10)$ and DAT-tg rats $(\mathrm{n}=10)$. All data are means \pm s.e.m. Asterisk $\left(^{*}\right)$ indicates significant difference to $w t$ rats, paragraph $(\$)$ indicates treatment effect $(\mathrm{p}<0.05)$. 
the dominant behavior observed upon chronic intermittent application of the DRD2/DRD3 agonist quinpirole, which in $w t$ rats induced compulsive checking behavior as previously reported ${ }^{14-16}$. Despite increased arousal and a tendency to anhedonia DAT-tg rats displayed intact sensorimotor gating, and scored normal in anxietyand depression-associated paradigms. All together this suggests that behavioral abnormalities in DAT-tg rats are largely restricted to repetitive behavior symptomatology.

Testing pharmacotherapy, we found that clonidine specifically reduced repetitive behavior in DAT-tg rats whereas the serotonin reuptake inhibitor (SSRI) fluoxetine did not selectively affect repetitive behavior in $D A T$ - $t g$ rats. As expected, fluoxetine decreased locomotion in both phenotypes ${ }^{17}$. Clonidine is known to alleviate tics in TS whereas SSRI agents have been shown to ameliorate repetitive symptoms in OCD but not in TS.

The potential utility of DAT-tg rats in the context of repetitive disorder research is further supported by the neurobiological investigations of this study. TS has previously been associated with increased and decreased dopamine receptor availability ${ }^{6,9,18,19}$ and dopamine contents ${ }^{5,18,20-22}$. We found that ubiquitously induced DAT overexpression induced a region specific pattern of up- and downregulation. DAT- $t g$ rats showed relative overexpression of DRD1 + DRD2 in the OFC, CPu and Nacc. This was further paralleled by increased striatal MAO enzymatic activity, previously linked to $\mathrm{TS}^{23}$. Increased $\mathrm{MAO}$ activity leads to increased dopamine turnover, resulting in decreased in dopamine levels. In contrast, DRD1 + DRD2 expressions were downregulated in the $\mathrm{mPFC}$, Thal and STN and dopamine contents were reduced in the Thal and STN, which suggests a reciprocal regulation of dopamine receptor expression and tissue dopamine contents ${ }^{24}$.

In TS patients, an altered balance between GABAergic cells and glutamatergic projections is associated with abnormal corticostriatal circuit activity ${ }^{2}$. This imbalance is thought to result from reduced numbers of GABAergic parvalbumin expressing $(\mathrm{PV}+)$ interneurons. In accordance with that DAT-tg displayed region-specific increments and decrements in GABAergic and glutamatergic contents in the corticostriatal circuit. Further, we found a reduction of $\mathrm{PV}+$ interneurons in DAT- $\mathrm{tg}$ rats as compared to $w t$ rats. In line with clinical data ${ }^{25,26}$, this reduction was restricted to the $\mathrm{CPu}$. Striatal $\mathrm{PV}+$ interneurons coordinate striatal activity by increasing medium spiny neurons' (MSN) firing threshold in response to cortical inputs ${ }^{25,27}$. Loss of PV + interneurons found in TS patients is suggested to lead to MSN hyperactivity ${ }^{25,26}$. Both MSN and PV+ cells express dopamine receptors and depending on the membrane-potential are susceptible to dopaminergic activation ${ }^{27,28}$. The excessive depolarized state of MSNs facilitates the effect of dopamine on MSNs, which further reinforces their hyperactivity. As such, abnormalities in the striatal PV+ interneuron and dopamine systems may together induce an excessive activation of the cortico-striato-thalamic circuit leading to repetitive behavior ${ }^{28,29}$.

Further linkages of DAT-tg to repetitive disorders were gained by studies into neuronal cellular and population activity. DAT-tg rats exhibited upregulation of c-Fos in the OFC. Increased OFC activity is observed in patients with repetitive disorders ${ }^{29,30}$. DAT-tg rats further displayed increased beta and gamma oscillations in the mPFC, Nacc and STN and increased alpha oscillation in the STN. Beta activity in the STN is associated with movement abnormalities and inversely regulated by mesostriatal dopamine ${ }^{31}$. Alpha and gamma activity has been associated with spontaneous tic exertion and $\mathrm{TS}^{32}$. In general, alterations in LFP oscillatory activity are proposed as biomarkers of dopamine dysfunction ${ }^{31}$ and neuro-psychiatric disorders ${ }^{33}$.

Ectopic DAT overexpression has previously been linked to neurotoxic events including oxidative stress and neuronal loss ${ }^{34-36}$. To explore whether DAT overexpression induced neuropathological changes in the $D A T$ - $t g$ rat, we measured the volume of the mPFC, the striatum and the hippocampus as these areas in the DAT-tg rat displayed both ectopic DAT expression and dopaminergic input but showed differential effects of DAT-overexpression on DA contents. MRI data displayed no atrophy in either the mPFC or the CPu but increased Hipp volumes in DAT-tg as compared to control rats. Increased Hipp volumes have been suggested to constitute a compensatory response in $\mathrm{TS}^{37}$. Further immunostaining of the neuron-specific marker (NeuN) revealed no difference between the phenotypes, which stresses the notion that ubiquitous overexpression of DAT does not induce neurotoxicity in the DAT-tg rat.

Our findings support the hypothesis that the DAT may constitute one important key component in repetitive pathophysiology and that DAT overexpression might be of relevance for further comprehension of neurobiological mechanisms underlying neuropsychiatric disorders.

\section{Experimental Procedures}

Rats. Rats were housed in a temperature- and humidity-controlled vivarium with a 12-h light dark cycle (lights on 06:00 a.m.) with food and water available ad libitum. The study was carried out in accordance with the European Communities Council Directive of 22th September 2010 (2010/63/EU) and after approval by the local ethic committees (Senate of Berlin and Regierungspräsidium Dresden). All efforts were made to reduce animal suffering and number of animals used.

Preparation of the construct. The pcDNA3-murine dopamine transporter ${ }^{38}$ (mDAT) cDNA-vector was kindly provided by Heinz Bönisch (Institute of Pharmacology and Toxicology, University of Bonn, Germany) (Fig. 1a). It contains the full coding region of the mDAT cDNA and has been cloned by PCR with a sense primer derived from the partial mDAT gene sequence and an antisense primer deduced from the rat DAT cDNA. In this construct the CMV promoter was replaced by the rat NSE promoter isolated from the pNSE-Ex4 vector comprising $2.6 \mathrm{~kb}$ of $5^{\prime}$-untranslated sequence plus exon 1 , intron 1 , and $6 \mathrm{bp}$ of exon 2 but not the ATG start codon of NSE. Sequencing was performed by the University of Calgary DNA Sequencing Laboratory to confirm the sequence of the construct. The construct consisting of NSE promoter, mDAT coding sequence, and bovine growth hormone polyadenylation sequence was excised from the pcDNA3 vector with NruI/NaeI, purified by agarose gel electrophoresis and gel extraction using the QIAquick gel extraction kit and used for microinjection. The NSE promoter was chosen for the expression of DAT to avoid probable unpredictable effects due to increment in monoamine in dopaminergic nerve endings. 
Generation of transgenic rats was conducted as reported previously ${ }^{39}$. Briefly, immature female Sprague-Dawley (SD) Hanover rats (28 to 35-day-old from Janvier Labs, France) were induced to superovulate by intraperitoneal (i.p.) injection of PMSG (15 IU, Intervet) and hCG (30 IU Sigma). Thereafter, rats were mated with fertile males and $24 \mathrm{~h}$ later sacrificed to collect fertilized eggs. The DNA construct was microinjected into the pronucleus of zygotes ${ }^{40,41}$. Eggs were cultured for two hours and the surviving DNA-injected zygotes were transferred into the oviducts of pseudopregnant SD recipients at the day the vaginal plug was detected. Integration of the transgene was determined by transgene-specific PCRs with genomic DNA isolated from tail biopsies of the offspring after weaning (Fig. 1b). Neurobiological and behavioral studies were conducted on adult male hemizygous DAT-transgenic rats (DAT-tg) ubiquitously overexpressing DAT in the corticostriatal and the associated networks. Wildtype $(w t)$ rats served as controls. Immunohistochemical staining of DAT expression was carried out for wt and DAT-tg rats (Fig. 1c).

Tissue processing. For Western blotting (WB), quantitative real time PCR (qPCR), and post mortem HPLC, and MAO activity assay, rats were decapitated and micropunches were taken bilaterally from $0.5-1 \mathrm{~mm}$ thick brain slices from the medial prefrontal cortex (mPFC), orbitofrontal cortex (OFC), thalamus (Thal), hippocampus (Hipp), nucleus accumbens (Nacc), caudate putamen $(\mathrm{CPu})$, globus pallidus $(\mathrm{GP})$ and subthalamic nucleus (STN) as described previously ${ }^{42}$. The total RNA and protein was extracted using the NucleoSpin RNA/Protein-Kit (Machery-Nagel, Düven, Germany). For immunostaining, rats were transcardially perfused, brains postfixed in $4 \%$ paraformaldehyde and cryosectioned in $40-\mu \mathrm{m}$ serial coronal frozen sections.

Western blotting. Protein concentrations were determined using a Nanodrop Spectrophotometer (peqlab) (UV $280 \mathrm{~nm}$ ). Samples (pooled Nacc and CPu specimen only) were loaded alongside Precision Plus Protein Kaleidoskope Standards (Bio-Rad), subjected to discontinuous electrophoresis on 10\% SDS-polyacrylamide gels and then transferred onto PVDF membranes (Roth) by electroblotting. Membranes were first incubated in SuperBlock T20 (TBS) Blocking Buffer (Lifetechnologies) at room temperature for 1 hour, and then incubated at $4{ }^{\circ} \mathrm{C}$ overnight with the primary antibodies: anti-DAT (1:200 dilution, Santa Cruz, sc-14002). A ß -actin antibody (1:800 dilution, Cell Signaling. 4967S) was used for internal control. Membranes were washed and incubated with horseradish peroxidase-conjugated secondary antibodies (1:5000 dilution, Amersham, ECL Rabbit IgG, HRP-linked whole antidbody: GE Healthcare Life Science NA934) at room temperature for one hour. For repeated analysis, membranes were stripped with Restore ${ }^{\mathrm{TM}}$ Plus Western Blot Stripping Buffer (Thermoscientifc). Detection of immunoreactive bands was conducted using the Western lighting plus enhanced chemiluminescence (ECL) reagent (PerkinElmer) on a cooled charge-coupled device camera (FLI Proline PL09000, PA, USA). Images were processed using the Image J software.

qPCR. RNA concentrations were determined using a Nanodrop Spectrophotometer (peqlab). cDNA was synthesized using the High Capacity RNA-to-cDNA Kit (Lifetechnologies). TaqMan qPCR was performed with StepOne Real-Time PCR System (Lifetechnologies) using TaqMan fast advanced master mix (Lifetechnologies). The following TaqMan Gene Expression assays (Lifetechnologies) were used: DAT (Rn00562224_m1), DRD1 (Rn 03062203_s1), and DRD2 (Rn01418275_m1). CT values were normalized to the house keeping gene GFAP (Rn00566603_m1, Lifetechnologies), fold change was calculated using the $\Delta \Delta \mathrm{CT}$ method.

Monoamine oxidase activity assay. For assessing monoamine oxidase (MAO) activity, CPu punches were homogenized by ultrasonication in $70 \mathrm{ul}$ assay buffer of a fluorometric assay kit (biovision K795-100). MAO activity was assessed according to the user manual.

Post mortem HPLC. Post mortem HPLC was conducted as described previously ${ }^{42}$. Dopamine and its metabolite DOPAC were separated on a column (ProntoSil 120-3-C18-SH; Bischoff Analysentechnik und -geräte $\mathrm{GmbH}$, Germany) and electrochemically detected (Chromsystems Instruments \& Chemicals GmbH, Germany). Glutamate and GABA were precolumn-derivatized with $o$-phthalaldehyde-2-mercaptoethanol, separated on a column (ProntoSil C18 ace-EPS) and detected by their fluorescence at $450 \mathrm{~nm}$ after excitation at $330 \mathrm{~nm}$.

Immunostaining. Free-floating sections were stained with antibodies against Ki67 (1:500, Novocastra, NCL-Ki67p), NeuN (1:5000, Millipore MAB377), DAT (1:50, Millipore AP1569P), Parvalbumin (PV+, 1:500, Antikörper-online, ABIN1742405), c-Fos (1:100, Santa Cruz, sc-52) and detected with goat-anti-rabbit biotinylated secondary antibodies (1:1000, Vector Laboratories, BA1000). For PV+ immunostaining, one-in-twelve series from the rostral-caudal extent of the mPFC, Hipp, CPu and GP and for Ki67 immunostaining one-in-twelve series from the Hipp and the subventricular zone (SVC) were analyzed. For c-Fos and NeuN immunostaining, the number of positive nuclei that fell within a $0.5 \times 0.5 \mathrm{~mm}$ area ( 2,5 objective) in the $\mathrm{mPFC}$, OFC, Thal, Hipp, $\mathrm{Nacc}, \mathrm{CPu}, \mathrm{GP}$ and STN was counted from one-in-twelve series sections from the rostral-caudal extent of the respective regions ${ }^{43}$. A representative picture of the DAT transporter was obtained.

MRI. MRI was performed using a 7 Tesla rodent scanner (Pharmascan 70/16, Bruker BioSpin,Ettlingen, Germany) and a ${ }^{1} \mathrm{H}$-RF quadratur-volume resonator with an inner diameter of $20 \mathrm{~mm}$ on ex vivo brains. Data acquisition and image processing were carried out with the Bruker software Paravision 5 . All brains had been perfused and snap frozen in methylbutan. 24 hours prior to the scan, all brains were placed in phosphate-buffered saline (PBS) and stored at $4{ }^{\circ} \mathrm{C}$, to allow for the defrosting of the brains. On the day of MRI acquisition, rat brains were placed in a $15 \mathrm{ml}$ Falcon tube containing PBS-with the anterior-posterior axis of the brain co lining with the long axis of the tube. For imaging the whole brain a T2-weighted 2D turbo spin-echo sequence was used (imaging parameters $\mathrm{TR} / \mathrm{TE}=5980.3 / 36 \mathrm{~ms}$, rare factor 8,4 averages, 50 axial slices with a slice thickness of $0.5 \mathrm{~mm}$, field 
of view of (FOV) $20.59 \times 20.59 \mathrm{~mm}$, matrix size $256 \times 256$ ). Brain structure volume was estimated as the mean magnitude of regions of interest (ROI) using ImageJ software.

Electrophysiology. Local field potentials (LFPs) were recorded under urethane anesthesia (1.2 g/kg i.p., Sigma Aldrich, Germany) as descried previously ${ }^{44}$. Monopolar recording electrodes (polyimide insulated stainless steel, $0.125 \mathrm{~mm}$, Plastics One, USA) were implanted ipsilaterally into the left mPFC, Nacc shell, and STN at the following coordinates with respect to bregma: $\mathrm{mPFC}: \mathrm{AP}=3.5, \mathrm{ML}=0.6, \mathrm{DV}=-3.4, \mathrm{Nacc}: \mathrm{AP}=1.2$, $\mathrm{ML}=1.8, \mathrm{DV}=-8.1, \mathrm{STN}: \mathrm{AP}=-3.6, \mathrm{ML}=2.5, \mathrm{DV}=-7.6^{45}$. Recordings were referenced against $1.2 \mathrm{~mm}$ steel screws affixed to the skull in close proximity to each recording electrode. Signals were bandpass filtered $(0.05 \mathrm{~Hz}-300 \mathrm{~Hz})$, amplified, sampled at $1 \mathrm{kHz}$ and digitized using a programmable neuronal data acquisition system (Omniplex, Plexon, Texas, USA). Recordings were conducted over a period of five hours. Offline data from the mPFC were inspected visually to identify and analyze epochs (40-50 s) of robust activated synchronization states (AS) reflecting signals of awake behaving rats ${ }^{46}$. The same time segments identified to show robust AS in the mPFC were also used for analysis of LFPs from the Nacc shell. Power spectral densities of the LFP data segments were calculated by employing the Fast Fourier Transform function (Spike 2 Version 6 data analysis software; Hanning Window $(1024 \mathrm{~ms}), 0.9766 \mathrm{~Hz}$ resolution). Frequency spectrum was divided into four EEG bands: theta $(4-8 \mathrm{~Hz})$, alpha $(8-12 \mathrm{~Hz})$, beta $(12-30 \mathrm{~Hz})$, gamma $(30-100 \mathrm{~Hz})$. Power spectra were normalized to total power between $103-147 \mathrm{~Hz}$ and $153-197 \mathrm{~Hz}$. Power was averaged across the specific frequency bands and further expressed in arbitrary units (a. u.). Correct electrode tip placements were histologically verified.

Behavioral analysis and drug treatment. Amphetamine-Induced stereotypy. Testing took place in individual testing boxes $(50 \times 50 \times 50)$ composed of 4 identical Plexiglas walls. Boxes were visually isolated from each other by an opaque screen. Experiments were performed over three consecutive days, during which animals were subjected to the three different dosages of d-amphetamine (i.p $0.5 \mathrm{mg} / \mathrm{kg}, 2.0 \mathrm{mg} / \mathrm{kg}$ or $5.0 \mathrm{mg} / \mathrm{kg}$, dissolved in $0.9 \%$ saline at a volume of $1.0 \mathrm{ml} / \mathrm{kg}$, Sigma Aldrich, Germany) in a cross over design. On testing days and prior to injection animals were habituated to the testing boxes for $20 \mathrm{~min}$. Following injection, animals were immediately placed back into the testing boxes and behavior was recorded for $120 \mathrm{~min}$. For analysis, the $120 \mathrm{~min}$ test was divided into 5-min segments and the most prominent behavior was scored for each segment. Behavioral scoring was based on an adapted version of the scoring protocol employed by Carter et al. ${ }^{47}$ dividing behavioral expression into (i) limited exploratory activity with discontinuous sniffing/grooming/rearing (no locomotion), (ii) constant exploratory activity with discontinuous sniffing/grooming/rearing (locomotion), (iii) continuous rearing (rearing), (iv) continuous sniffing (sniffing), (v) continuous biting, gnawing or licking (oral stereotypy), (vi) continuous head swaying/head bobbing (head movements).

Amphetamine and clonidine/fluoxetine treatment. Testing took place in testing boxes as described above. Experiments were performed over two testing days $72 \mathrm{~h}$ apart, during which animals were randomly assigned to treatment (clonidine/fluoxetine) or control (saline) conditions in a cross over design. On both testing days, all animals were initially habituated to testing boxes for $20 \mathrm{~min}$ after which they were injected with amphetamine $(2.0 \mathrm{mg} / \mathrm{kg}$, dissolved in $0.9 \%$ saline at a volume of $1.0 \mathrm{ml} / \mathrm{kg}$, Sigma Aldrich, Germany), placed back into the testing boxes and video recorded. $50 \mathrm{~min}$ after amphetamine injection, animals were injected with clonidine (i.p $0.01 \mathrm{mg} / \mathrm{kg}$, dissolved in $0.9 \%$ saline at a volume of $10 \mathrm{ml} / \mathrm{kg}$, Sigma-Aldrich, Germany), fluoxetine $(20 \mathrm{mg} / \mathrm{kg}$, dissolved in $0.9 \%$ saline at a volume of $1.0 \mathrm{ml} / \mathrm{kg}$, Hexal, Germany) or saline after which they were placed back into the testing boxes. Behavior was analyzed for the period of most prominent expression of oral stereotypy in drug-free conditions, i.e. $80-120 \mathrm{~min}$ post amphetamine application. For analysis, the $40 \mathrm{~min}$ test period was divided into 5-min segments and the most prominent behavior was scored for each segment as described above.

Quinpirole induced repetitive behavior. Rats treated chronically with the dopamine D2/D3 receptor agonist quinpirole (QNP) develop compulsive-like behaviors that resemble compulsive checking behavior of OCD patients ${ }^{15}$. Rats were injected subcutaneously twice weekly for a total of 10 injections with either saline or QNP hydrochloride $\left(0.5 \mathrm{mg} / \mathrm{kg}\right.$ body weight, $0.5 \mathrm{mg} / \mathrm{ml} 0.9 \% \mathrm{NaCl}$, Sigma ${ }^{\circledR}$ Aldrich, Germany). Fifteen minutes after each injection, animals were placed in an open field that consisted of a glass table $(140 \times 140$ and $20 \mathrm{~cm}$ high) subdivided into 25 rectangles (locales) and equipped with 4 plexiglas boxes at fixed locations. The $10^{\text {th }}$ session, when QNP treated rats are known to display compulsive checking behavior was videotaped and analyzed using tracking software (VideoMot 2 system, TSE, Bad Homburg, Germany). The following measures were assessed: (i) total distance traveled, (ii) total time of activity/inactivity, (iii) frequency of stops at each open field locale, (iv) mean duration of return time to a given locale, (v) mean stop duration at a given locale, (vi) total duration of stops at a given locale. The locale with the highest total duration of stops was individually defined as the home base and compulsive checking behavior was analyzed with reference to the HB. Compulsive checking is present if the rat meets the following three criteria: it returns to HB excessively often, excessively rapidly, and visits less places before returning to the HB. As repeated administration of QNP increases locomotion and since checking behavior requires locomotion, arithmetic was applied allowing the assessment of checking behavior relatively independent from locomotion. Specifically, for each rat individually, the expected rate of return to a locale was calculated by dividing the total number of visits in a session by the number of locales visited. Next, the ratio of observed to expected $\mathrm{HB}$ visits was calculated by dividing the number of visits to the $\mathrm{HB}$ with the expected rate of return to a locale. Additionally, the total time spent on grooming/oral stereotypy on the $10^{\text {th }}$ test day was calculated. 
Startle stress response. Animals were exposed to unpredictable acoustic stimulus to investigate the effect of stressor-exposure on repetitive behaviour. Animals were placed in the chambers used for prepulse inhibition (PPI) test. The plastic enclosure used to restrain the rats during PPI testing was removed. The door was left open and a piece of clear Plexiglas was placed in front of the opening of the chamber to prevent the rats from escaping. Rats were acclimated to the box for $10 \mathrm{~min}$, then a PPI protocol was initiated and run for $10 \mathrm{~min}$, thereafter rats were left undisturbed for further $10 \mathrm{~min}^{12}$. The process was recorded and scored on playbacks. The total time spent on grooming as well as the number of whole body shakes during the $20 \mathrm{~min}$ after PPI protocol initiation was analyzed.

Prepulse inhibition of an acoustic startle response. Acoustic startle response (ASR) and PPI of the ASR was assessed using a standard startle chamber (SR-lab, San Diego Instruments). An adapted version of the general SR-LAB startle response user manual was applied. Animals were exposed to a $5 \mathrm{~min}$ acclimatization phase of white noise at $65 \mathrm{~dB}$, followed by 5 initial startle stimuli $(120 \mathrm{~dB}$, each presented for $40 \mathrm{~ms})$. The test session was pseudorandomized and composed of 40 startle stimuli presented either alone ( $120 \mathrm{~dB}$ for $40 \mathrm{msc}$ ), or proceeded by a pre-pulse of either 69,73 or $81 \mathrm{~dB}$ for $20 \mathrm{~ms}, 100 \mathrm{~ms}$ before the startle. Each pulse or pre-pulse trial was separated by inter-trial intervals of a randomized duration ranging from 15-30 seconds, during which white background noise was presented $(65 \mathrm{~dB})$, leading to a total testing time of approximately $40 \mathrm{~min}$. The animals' startle reaction to the stimuli alone and to the pre-pulse trials was measured for $100 \mathrm{~ms}$ following the stimulus and amplitude as well as percentage decrease in startle response with pre-pulses (pre-pulse inhibition) was estimated ${ }^{41}$.

Elevated plus maze. Animals were placed in the center of an elevated plus maze (EPM, $42 \times 42 \mathrm{~cm}$, arm width: $23 \mathrm{~cm}$ ), composed of two closed and two open arms. The animals were allowed to freely explore the maze for $5 \mathrm{~min}$, while behavior was recorded via a web camera. The total time spent on open arms (OA, with both frontand hind paws placed on the arm) was determined ${ }^{48}$.

Forced swim test. Animals were conditioned to water-filled glass cylinders (depth of $30 \mathrm{~cm}, 25^{\circ} \mathrm{C}$ ) for $15 \mathrm{~min}$ $24 \mathrm{~h}$ prior to testing. The cylinders were visually isolated from each other by an opaque screen. On testing day, animals were placed in the cylinders for $5 \mathrm{~min}$ and behavior was recorded via a web camera. For behavioral analyses, the 5 min test was divided into 5-second segments and the most predominant behavior was determined per segment (struggling, swimming and floating behaviour) ${ }^{48}$.

Sucrose consumption test. $48 \mathrm{~h}$ prior to testing, animals were habituated to the individual testing cages and bottles (containing water). $24 \mathrm{~h}$ thereafter, animals were habituated to the sweetend condensed milk (Nestlé, Milchmädchen gezuckerte Kondensmilch, (1:3)) for $30 \mathrm{~min}$ in their home cage and subsequently food restricted until time of testing ( $15 \mathrm{~g}$ per animal). On the day of testing, animals were placed in the individual cages with free access to the sweetend condensed milk for $15 \mathrm{~min}$. Bottles were weighed before and after testing. The amount of sweetend condensed milk consumed normalized to individual body weight was calculated ${ }^{48}$.

Blinding. Throughout the experiments best possible blinding was conducted. For video tracking during behavioral testing, animals were number-coded such that the experimenter was blinded to phenotype and treatment condition during later video analysis. The same system was applied to neurobiological analysis.

Statistical analysis. Data are shown as means \pm s.e.m. We used Student's t test to calculate significant differences between wt and DAT-tg rats. We used two-way ANOVA with the factors phenotype $(w t, D A T+/)$ and treatment (saline, QNP) for behavioral analysis of QNP induced repetitive behavior, and two-way ANOVA with repeated measure with the factors phenotype ( $w t, D A T-t g$ ) and treatment (saline, clonidine/fluoxetine) for drug experiments followed by Holm-Sidak post hoc test if applicable. Significance was set at $\mathrm{P}<0.05$.

\section{References}

1. Matthysse, S. Animal models in psychiatric research. Prog Brain Res 65, 259-70 (1986).

2. Leckman, J. F., Bloch, M. H., Smith, M. E., Larabi, D. \& Hampson, M. Neurobiological substrates of Tourette's disorder. J Child Adolesc Psychopharmacol 20, 237-247 (2010).

3. Singer, H. S. \& Walkup, J. T. Tourette Syndrome and Other Tic Disorders Diagnosis, Pathophysiology, and Treatment. Medicine 70, 15-32 (1991)

4. Ernst, M. et al. High presynaptic dopaminergic activity in children with Tourette's disorder. J. Am. Acad. Child Adolesc. Psychiatry 38, 86-94 (1999).

5. Singer, H. S. et al. Elevated intrasynaptic dopamine release in Tourette's syndrome measured by PET. Am J Psych. 159, 1329-36 (2002).

6. Cheon, K. A. et al. Dopamine transporter density of the basal ganglia assessed with [123 I] IPT SPECT in drug-naive children with Tourette's disorder. Psychiatry Res Neuroimaging 130, 85-95 (2004).

7. Serra-Mestres, J. et al. Dopamine transporter binding in Gilles de la Tourette syndrome: a [123I] FP-CIT/SPECT study. Acta Psychiatric Scand 109, 140-6 (2004).

8. Paschou, P. The genetic basis of Gilles de la Tourette Syndrome. Neurosci Biobehav Rev 37, 1026-1039 (2013).

9. Denys, D. et al. Dopaminergic activity in Tourette syndrome and obsessive-compulsive disorder. Eur Neuropsychopharmacol 23, 1423-1431 (2013).

10. Kossoff, E. H. \& Singer, H. S. Tourette syndrome: clinical characteristics and current management strategies. Paediatr Drugs. 3, 355-63. (2001)

11. McNaught, K. S. P. \& Mink, J. W. Advances in understanding and treatment of Tourette syndrome. Nat Rev Neurol 7, 667-676 (2011).

12. Xu, M. et al. Targeted ablation of cholinergic interneurons in the dorsolateral striatum produces behavioral manifestations of Tourette syndrome. PNAS 112, 893-898 (2015). 
13. Antoniou, K., Kafetzopoulos, E., Papadopoulou-Daifoti, Z., Hyphantis, T. \& Marselos, M. D-amphetamine, cocaine and caffeine: a comparative study of acute effects on locomotor activity and behavioural patterns in rats. Neurosci Biobehav Rev 23, 189-196 (1998).

14. Winter, C. et al. High frequency stimulation and temporary inactivation of the subthalamic nucleus reduce quinpirole- induced compulsive checking behavior in rats. Exp Neurol 210, 217-28 (2008).

15. Mundt, A. et al. High-frequency stimulation of the nucleus accumbens core and shell reduces quinpirole-induced compulsive checking in rats. Eur J Neurosci 29, 2401-2412 (2009).

16. Djodari-Irani, A. et al. Activity modulation of the globus pallidus and the nucleus entopeduncularis affects compulsive checking in rats. Behav Brain Res 219, 149-58 (2011).

17. Pic-Taylor, A. et al. Behavioural and neurotoxic effects of ayahuasca infusion (Banisteriopsis caapi and Psychotria viridis) in female Wistar rats. Behav Processes 118, 102-110(2015).

18. Wong, D. F. et al. Mechanisms of dopaminergic and serotonergic neurotransmission in Tourette syndrome: clues from an in vivo neurochemistry study with PET. Neuropsychopharmacology 33, 1239-51 (2008).

19. Gilbert, D. L., Christian, B. T., Gelfand, M. J., Shi, B., Mantil, J. \& Sallee, F. R. Altered mesolimbocortical and thalamic dopamine in Tourette syndrome. Neurology 67, 1695-1697 (2006).

20. Singer, H. S., Butler, I. J., Tune, L. E., Seifert, W. E. Jr. \& Coyle, J. T. Dopaminergic dsyfunction in Tourette syndrome. Ann Neurol 12, 361-6 (1982).

21. Butler, I. J., Koslow, S. H., Seifert, W. E. Jr, Caprioli, R. M. \& Singer, H. S. Biogenic amine metabolism in Tourette syndrome. Ann Neurol. 6, 37-9 (1979).

22. Cohen, D. J., Shaywitz, B. A., Caparulo, B., Young, J. G. \& Bowers, M. B. Jr. Chronic, multiple tics of Gilles de la Tourette's disease. CSF acid monoamine metabolites after probenecid administration. Arch Gen Psychiatry. 35, 245-50 (1978).

23. Shapiro, A. K., Baron, M., Shapiro, E. \& Levitt, M. Enzyme activity in Tourette's syndrome. Arch Neurol 41, 282-285 (1984).

24. Segawa, M. Neurophysiology of Tourette's syndrome: pathophysiological considerations. Brain Dev 25, S62-S69 (2003).

25. Kalanithi, P. S. et al. Altered parvalbumin-positive neuron distribution in basal ganglia of individuals with Tourette syndrome. PNAS 102, 13307-13312 (2005).

26. Kataoka, Y. et al. Decreased number of parvalbumin and cholinergic interneurons in the striatum of individuals with Tourette syndrome. J Comp Neurol 518, 277-291 (2010).

27. Koós, T. \& Tepper, J. M. Inhibitory control of neostriatal projection neurons by GABAergic interneurons. Nat Neurosci 2, 467-472 (1999).

28. Singer, H. S. \& Minzer, K. Neurobiology of Tourette's syndrome: concepts of neuroanatomic localization and neurochemical abnormalities. Brain Dev 25, S70-S84 (2003).

29. Mink, J. W. Basal ganglia dysfunction in Tourette's syndrome: a new hypothesis. Pediatr Neurol 25, 190-198. (2001).

30. Menzies, L. et al. Integrating evidence from neuroimaging and neuropsychological studies of obsessive-compulsive disorder: the orbitofronto-striatal model revisited. Neurosci Biobehav Rev 32, 525-549 (2008).

31. Mallet, N. et al. Disrupted dopamine transmission and the emergence of exaggerated beta oscillations in subthalamic nucleus and cerebral cortex. J Neurosci 28, 4795-4806 (2008).

32. Hong, H. J. et al. Increased frontomotor oscillations during tic suppression in children with Tourette syndrome. J Child Neurol 28, 615-624 (2013)

33. Neumann, W. J. et al. Different patterns of local field potentials from limbic DBS targets in patients with major depressive and obsessive compulsive disorder. Mol Psych. 19, 1186-92 (2014).

34. Masoud, S. T. et al. "Increased expression of the dopamine transporter leads to loss of dopamine neurons, oxidative stress and 1-DOPA reversible motor deficits". Neurobiol dis. 74, 66-75 (2015).

35. Fazeli, G. et al. "The role of Dopamine Transporter in Dopamine-Induced DNA Damage". Brain Pathol 21(3), 237-248 (2011).

36. Chen, L. et al. "Unregulated cytosolic dopamine causes neurodegeneration associated with oxidative stress in mice". J. Neurosci 28(2), 425-433 (2008).

37. Peterson, B. S. et al. Morphologic features of the amygdala and hippocampus in children and adults with Tourette syndrome. Arch Gen Psychiatry 64, 1281-1291 (2007).

38. Brüss, M., Wieland, A. \& Bönisch, H. Molecular cloning and functional expression of the mouse dopamine transporter. J Neural Transm 106, 657-662 (1999).

39. Popova, E., Bader, M. \& Krivokharchenko, A. Strain differences in superovulatory response, embryo development and efficiency of transgenic rat production. Transgenic Res 14, 729-738 (2005).

40. Forss-Petter, S. et al. Transgenic mice expressing $\beta$-galactosidase in mature neurons under neuron-specific enolase promoter control. Neuron 5, 187-197 (1990)

41. Mucke, L. et al. Synaptotrophic effects of human amyloid $\beta$ protein precursors in the cortex of transgenic mice. Brain Res 666, 151-167 (1994)

42. Hadar, R. et al. Using a maternal immune stimulation model of schizophrenia to study behavioral and neurobiological alterations over the developmental course. Schiz Res 166, 238-247 (2015).

43. Carson, D. S. et al. Systemically administered oxytocin decreases methamphetamine activation of the subthalamic nucleus and accumbens core and stimulates oxytocinergic neurons in the hypothalamus. Addict Biol 15, 448-463 (2010).

44. Voget, M. et al. Altered local field potential activity and serotonergic neurotransmission are further characteristics of the Flinders sensitive line rat model of depression. Behav Brain Res 291, 299-305(2015).

45. Paxinos, G. \& Watson, C., A stereotaxic atlas of the rat brain. New York: Academic (1998).

46. Magill, P. J. et al. Changes in functional connectivity within the rat striatopallidal axis during global brain activation in vivo. J Neurosci 26, 6318-6329 (2006).

47. Carter, C. J. \& Pycock, C. J. The effects of 5, 7-dihydroxytryptamine lesions of extrapyramidal and mesolimbic sites on spontaneous motor behaviour, and amphetamine-induced stereotypy. Naunyn-Schmiedeberg's Arch Pharmacol 308, 51-54 (1979).

48. Edemann-Callesen, H. et al. Medial Forebrain Bundle Deep Brain Stimulation has Symptom-specific Anti-depressant Effects in Rats and as Opposed to Ventromedial Prefrontal Cortex Stimulation Interacts With the Reward System. Brain Stim 8, 714-23 (2015).

\section{Acknowledgements}

We thank Renate Winter, Christiane Kölske, Jennifer Altschüler, Sarina Sabrina Richter and Christian Böttcher for excellent technical support, Dirk Megow and Susanne Müller for profound support on Western blotting and MRI studies. The study was funded by Else Kröner-Fresenius Foundation (Grant 2008_A132) and the Federal Ministry of Education and Research, Germany (Grant 01EW1409 (EraNet Neuron framework) and Grant 01EE1403A (GCBS)). RH, FW, MV, and JP partly were or are financed by the German Research Foundation (Grant PAK 591: WI 2140/2-1, Grant KFO247: WI 2140/1-1/2 and Grant FOR1336-2: C4). 


\section{Author Contributions}

R.H. contributed to experimental design, data analysis and interpretation and wrote the ms. H.E.-C. conducted behavioral and MRI experiments, contributed to behavioral data analysis and writing the ms. C.R. conducted molecular biological and contributed to biochemical investigations. F.W. conducted immunohistochemical and biochemical and contributed to molecular biological investigations. M.V. conducted electrophysiological experiments, contributed to immunohistochemical investigations and writing the ms. E.P. generated transgenic rats. R.S. supervised biochemical investigations. Y.A. contributed to electrophysiological investigation and data analysis. J.P. supervised Western blotting and C.v.R. electrophysiological experiments. I.P. generated the construct. M.B. supervised generation of construct and transgenic rats. C.W. conceived the study, designed experiments, conducted data analysis and interpretation and wrote the $\mathrm{ms}$.

\section{Additional Information}

Competing financial interests: The authors declare no competing financial interests.

How to cite this article: Hadar, R. et al. Rats overexpressing the dopamine transporter display behavioral and neurobiological abnormalities with relevance to repetitive disorders. Sci. Rep. 6, 39145; doi: 10.1038/srep39145 (2016).

Publisher's note: Springer Nature remains neutral with regard to jurisdictional claims in published maps and institutional affiliations.

(c) (i) This work is licensed under a Creative Commons Attribution 4.0 International License. The images or other third party material in this article are included in the article's Creative Commons license, unless indicated otherwise in the credit line; if the material is not included under the Creative Commons license, users will need to obtain permission from the license holder to reproduce the material. To view a copy of this license, visit http://creativecommons.org/licenses/by/4.0/

(C) The Author(s) 2016 